\title{
The effects of simulated vision impairments on the cone of gaze
}

\author{
Heiko Hecht $^{1}$ • Jenny Hörichs ${ }^{1} \cdot$ Sarah Sheldon $^{2,3} \cdot$ Jessilin Quint $^{4} \cdot$ Alex Bowers $^{2,3}$
}

Published online: 28 May 2015

(C) The Psychonomic Society, Inc. 2015

\begin{abstract}
Detecting the gaze direction of others is critical for many social interactions. We explored factors that may make the perception of mutual gaze more difficult, including the degradation of the stimulus and simulated vision impairment. To what extent do these factors affect the complex assessment of mutual gaze? Using an interactive virtual head whose eye direction could be manipulated by the subject, we conducted two experiments to assess the effects of simulated vision impairments on mutual gaze. Healthy subjects had to demarcate the center and the edges of the cone of gaze - that is, the range of gaze directions that are accepted for mutual gaze. When vision was impaired by adding a semitransparent white contrast reduction mask to the display (Exp. 1), judgments became more variable and more influenced by the head direction (indicative of a compensation strategy). When refractive blur was added (Exp. 1), the gaze cone shrank from $12.9^{\circ}$ (no blur) to $11.3^{\circ}$ (3-diopter lens), which cannot be explained by a lowlevel process but might reflect a tightening of the criterion for mutual gaze as a response to the increased uncertainty. However, the overall effects of the impairments were relatively modest. Elderly subjects (Exp. 2) produced more variability but did not differ qualitatively from the younger subjects. In the face of artificial vision impairments, compensation
\end{abstract}

Heiko Hecht

hecht@uni-mainz.de

1 Department of Psychology, Johannes Gutenberg-Universität Mainz, D-55099 Mainz, Germany

2 Schepens Eye Research Institute, Massachusetts Eye and Ear Infirmary, Boston, MA, USA

3 Department of Ophthalmology, Harvard Medical School, Boston, MA, USA

4 Indiana University School of Optometry, Bloomington, IN, USA mechanisms and criterion changes allow us to perform better in mutual gaze perception than would be predicted by a simple extrapolation from the losses in basic visual acuity and contrast sensitivity.

Keywords Gaze perception - Eye contact $\cdot$ Head orientation . Gaze cone $\cdot$ Central vision loss $\cdot$ Cataracts

The gaze of others is an important signal that regulates social interactions (Kleinke, 1986). The structure of the human eye, with high contrast between the iris and the white sclera, is especially well suited to signal gaze direction to another person (Ando, 2002), and our visual system seems to be particularly capable of processing gaze-related features. Rather surprisingly, however, human observers accept a wide range of gaze directions as constituting mutual gaze, suggesting that the precision of the sensory system is not being fully exploited (Gibson \& Pick, 1963). More precisely, the sum of the gaze directions that constitute mutual gaze should be thought of as a cone centered on the interpupillary point. This gaze cone is about $10^{\circ}$ wide. We have established a psychophysical method to measure the direction and width of the gaze cone (for details, see Gamer \& Hecht, 2007). In the centering task, in which the direction of the gaze cone is obtained, the subject is asked to adjust the eyes of the looker (i.e., the virtual head) via button presses on the keypad until the looker gazes straight (directly) toward the subject. On each trial, the initial gaze direction of the looker is clearly toward the left or the right. In the decentering task, which assesses the width of the gaze cone, the subject is asked to guide the looker's eyes either to the left or to the right until the virtual head is about to subjectively stop gazing at the subject. In the centering task, the direction of the gaze cone is expressed as the average gaze deviation from the subject's straight ahead (in degrees). The 
width of the gaze cone is expressed as the angular difference between the leftward and rightward boundaries of the sector within which gaze directions are considered as looking at the subject. Note that the direction and width of the gaze cone do not depend on the eye direction alone, but also on other parameters - for instance, the direction of the virtual head with respect to the eyes (Gamer \& Hecht, 2007).

\section{Visual impairments}

Thus far, the gaze cone has typically been assessed in young observers with normal visual acuity, and in good lighting conditions. We know that the gaze cones measured with the virtual head are similar to - albeit somewhat larger than - those obtained with a human looker, and we know that extraneous factors, such as the number of onlookers also looking toward the subject, do affect the cone of gaze (Gamer, Hecht, Seipp, \& Hiller, 2011; Hecht, Weiland, \& Boyarskaya, 2011). Here we explored other extraneous factors that may make the perception of mutual gaze more difficult. Specifically, we conducted two experiments to evaluate the effects of stimulus degradations and simulated vision impairments on mutual gaze perception in younger and older normally sighted observers.

At first sight, it might be thought that vision impairment should negatively affect the cone of gaze. However, although sufficient visual acuity and contrast sensitivity are prerequisites for the detection of gaze direction, the latter involves higher-level visual processes and judgment. If all other parameters remain unchanged, then decrements in acuity should reduce performance. However, if the higher-level processes change in response to the impairment, its effects will be much harder to predict. It is not clear to what extent the perceptual system might attempt to cope with the impairment. This could be done by means of compensation strategies such as increasing the effort dedicated to the task or searching for other perceptual cues that are correlated with the missing information, but are still available despite the impairment. In addition to effort and information substitution, it is also conceivable that the criterion used for the detection of mutual gaze might be changed. Thus, the resultant perception of gaze direction need not necessarily suffer with visual impairment; it may or may not be robust in the face of moderate stimulus degradation. We conducted two experiments to assess the effects of vision impairments.

\section{Experiment 1: Simulated vision impairments in young observers}

In the first experiment, we tested the hypothesis that degrading the appearance of a virtual head would interfere with mutual gaze perception. Specifically, we predicted that as the level of degradation increased, mutual gaze performance would become more variable and subjects would be more willing to accept a given gaze as being directed toward them (i.e., the gaze cone width would increase). The visibility of the head was reduced in two ways: A virtual semitransparent white contrast reduction mask was used to decrease the contrast and the high-spatial-frequency content of the stimulus, as is illustrated in Fig. 1. The other method of reducing visibility was the introduction of dioptric blur, which reduced the visual acuity of the subjects, simulating the situation of people with myopia (short-sightedness) who were not wearing their glasses or contact lenses.

\section{Method}

The experiment was approved by the Ethics Committee of the Johannes Gutenberg-University Mainz in Germany, where the data were collected. Voluntary, written informed consent was obtained from all subjects after a full explanation of the study procedures.

Subjects A group of 24 students volunteered for the experiment (11 men, 13 women; $19-36$ years of age, $M=24.15$, $S D$ $=4.53)$. About half of them received partial course credit for

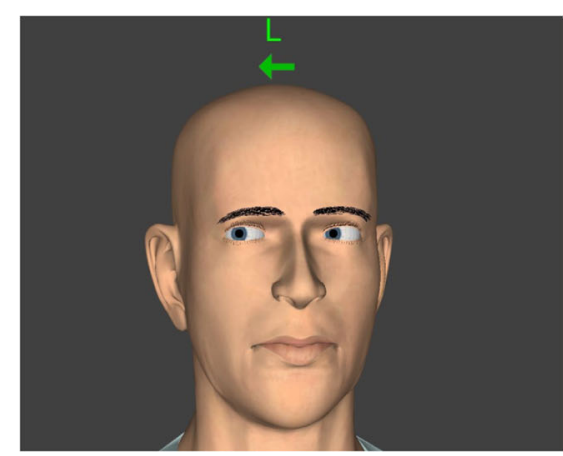

Fig. 1 The virtual head as seen without the semitransparent mask (100\% transparency, left panel) and with the virtual mask (15\% transparency setting, right panel). In this example, the head is rotated $8^{\circ}$ to its left, such that the nose points to the subject's right and the virtual eyes are clearly averted to the subject's left. A green letter and arrow indicated the task for each trial (in this case, to decenter the eyes to the left) 
their participation. Most of them were psychology students at the University of Mainz, and all had normal or corrected-tonormal visual acuity and contrast sensitivity.

Apparatus and stimuli A natural-looking human head was rendered using the 3-D software Vizard (Version 3.18, 2010) on a Dell Precision 380 computer. It was displayed on a 17-in. flat screen with a resolution of $1,280 \times 1,024$ pixels. The effective color depth (Graphics card nVIDIA Quadro FX3500) was $24+8$ bits ( 8 per channel +8 alpha). The virtual head and the eyes were intrinsically $3-\mathrm{D}$. We had previously presented the head in stereoscopic 3-D and found that the 2-D viewing of this stimulus had little effect on the gaze cone (Gamer \& Hecht, 2007). Thus, we used the 2-D view here. The width of the virtual head was $16.5 \mathrm{~cm}$ and the height $25.8 \mathrm{~cm}$, with an interpupillary distance of $6.4 \mathrm{~cm}$. This screen size of the virtual head (looker) approximately equaled the visual angle of an adult human head when viewed at $0.5 \mathrm{~m}$. The eyes were rendered independently and could be rotated interactively with a trackball. Their movement was confined to the horizontal plane, such that the line of sight was level with the subject's eye height. The vergence of the eyes of the virtual head was set such that their gaze always converged at the subject's eye plane. That is, when looking straight ahead, the looker's eyes converged at the subject's interpupillary point. When looking to the side, the virtual eyes would converge on the subject's eye plane to the left or the right. The subject was seated $0.5 \mathrm{~m}$ from the screen in a heightadjustable chair combined with a chinrest, which ensured that the subject's eye height was $1 \mathrm{~m}$ at all times. The eye height of the virtual head was adjusted to the same height by mounting the flat screen on a table.

Simulated impairments In the dioptric-blur condition, subjects wore an optometrist's trial frame, into which lenses of different dioptric values $(0,+1.5$, and +3 diopters $)$ were inserted. For long-distance vision, the 1.5- and 3-diopter lenses would be expected to create about 1.5 and 3 diopters of myopia, respectively. However, at the $0.5-\mathrm{m}$ test distance (where 2 diopters of accommodation is required for a clear image), it was expected that the 1.5-diopter lens would create only a subtle level of blur, and that the 3-diopter lens would create more noticeable blur, equivalent to about 1 diopter of uncorrected myopia. In the mask condition, a virtual semitransparent white mask was placed in front of the virtual head, producing transparency of $15 \%$, which reduced the stimulus contrast. The mask was applied to the entire image; it appeared as if fog had set in around the virtual head or as if the head were being viewed through ground glass (Fig. 1). In order to have an independent measure of the effects of the dioptric blur and the mask on visual acuity and contrast sensitivity, we recruited two groups ( $N=6$ and 9) of additional subjects from the same student population (average ages 28 and 26 years, respectively). Visual acuity was measured using the Landolt $\mathrm{C}$ set of the Freiburg Acuity Test (FrACT, http:// michaelbach.de/fract/html; Bach, 1996, 2006), presented on a high-resolution monitor $(52 \times 29.4 \mathrm{~cm}$, resolution $1,920 \times$ 1,080 , luminance $8.5 \mathrm{~cd} / \mathrm{m}^{2}$ ) in a darkened room. Contrast sensitivity was measured with a custom test based on the Pelli-Robson chart (Pelli, Robson, \& Wilkins, 1988) with letters that subtended $0.5^{\circ} \times 0.5^{\circ}$ of visual angle. The chart included eight lines, with two groups of three letters on each line. The contrast of each triplet was reduced in steps of 0.15 $\log$ units, such that the $\log$ contrast sensitivity varied from 0 . 00 to 2.25 ( $\log C S$ Weber). The luminance of the white background was $85 \mathrm{~cd} / \mathrm{m}^{2}$ in the no-mask condition.

To quantify the effects of the optical blur and the virtual mask on long-distance vision, the first group of additional subjects completed visual acuity and contrast sensitivity measures at a 4-m test distance. As expected, both the 1.5- and 3diopter blurs and the mask substantially reduced visual acuity and contrast sensitivity (Figs. 2a and c, respectively). The visual acuity measures were not limited by monitor resolution at this distance. The second group of additional subjects completed vision measures at the $0.5-\mathrm{m}$ test distance used in the experimental gaze cone measurements. At this short distance, the visual acuity measures were limited by the smallest letter size that could be displayed, which was about $0.3 \log$ MAR (the approximate resolution limit of the monitor). Therefore, visual acuity without simulated impairments was limited to $0.3 \log$ MAR (20/40) at $0.5 \mathrm{~m}$ (Fig. 2b), as compared to about $-0.1 \log$ MAR (20/15) at $4 \mathrm{~m}$ (Fig. 2a). The 1.5-diopter blur had no effect on either visual acuity or contrast sensitivity at $0.5 \mathrm{~m}$ (Figs. 2b and d). By comparison, the 3-diopter blur reduced both visual acuity and contrast sensitivity. The mask had relatively little effect on visual acuity at 1.5 diopters of blur, but it reduced acuity at 3 diopters of blur (Fig. 2b) and substantially reduced contrast sensitivity in all viewing conditions (Fig. 2d).

Design and procedure Three factors were varied in a repeated measures design: Mask (two levels: transparency $100 \%$ and $15 \%$ ), Dioptric Blur (three levels: 0, 1.5, and 3.0 diopters), and Head Orientation (three levels). The virtual head either faced directly at the subject or was rotated around a vertical axis placed through a point between the eyes. In the latter case, the head rotation was $8^{\circ}$ to its right (yaw clockwise), resulting in the nose pointing to the subject's left (Fig. 1), or the head rotation was $8^{\circ}$ to the left (yaw counterclockwise), resulting in the nose pointing to the subject's right. Note that the eyes were not always aligned with the head, such that head orientation was independent of gaze direction. Subjects sat in a chair adjusted to produce the desired eye height of $1 \mathrm{~m}$. A letter and arrow on the screen indicated which one of the four adjustment tasks was to be completed (Fig. 1). Centering was called for if the virtual eyes initially gazed in a 
a

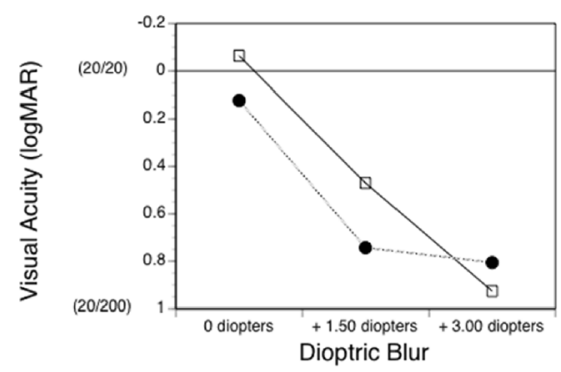

b

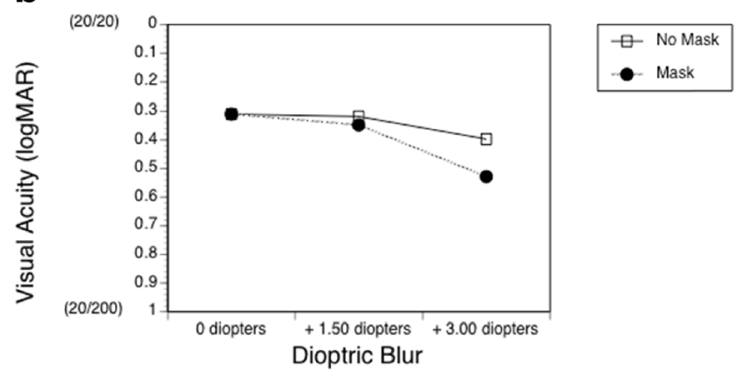

Fig. 2 (a, b) Landolt visual acuity thresholds without (empty squares) and with (black circles) the semitransparent mask for each of the three levels of dioptric blur, measured at viewing distances of (a) $4 \mathrm{~m}$ and (b) $0.5 \mathrm{~m}$. Note that at the short viewing distance, visual acuity threshold measurements were limited by the smallest letter size that could be displayed (about $0.3 \log$ MAR). For reference, Snellen acuities are given

direction between $7^{\circ}$ and $9^{\circ}$ to the left or $-7^{\circ}$ and $-9^{\circ}$ to the right of the subject. The values were randomly varied within this range in order to avoid a constant starting position and at the same time to leave sufficient room for adjustment. We instructed the subject to adjust the eyes of the virtual head such that it gazed directly at him or her. To do this, the subject rotated a trackball to move the gaze and was told to go back and forth, if needed, to home in on the setting that corresponded best to a direct gaze. Decentering was called for whenever the virtual head initially gazed directly at the subject, who was instructed to rotate the head's eyes either to the left or to the right until the virtual head was about to stop gazing at the subject. The adjustment was accomplished with a maximal accuracy of $0.1^{\circ}$ by using the trackball. Subjects were encouraged to find the gaze direction that best specified the outer boundary of eye contact. The order of all centering and decentering trials was randomly determined for each subject, and subjects pressed a button when they were satisfied with their setting. No time limit was specified.

All factors were fully crossed and performed together with each of four instructions (centering from an initial leftward/ rightward gaze, decentering to the left or the right). Thus, each subject performed a total of 72 trials (based on three levels of dioptric blur, two levels of mask, three head orientations, and four instructions). The three dioptric-blur and the two mask

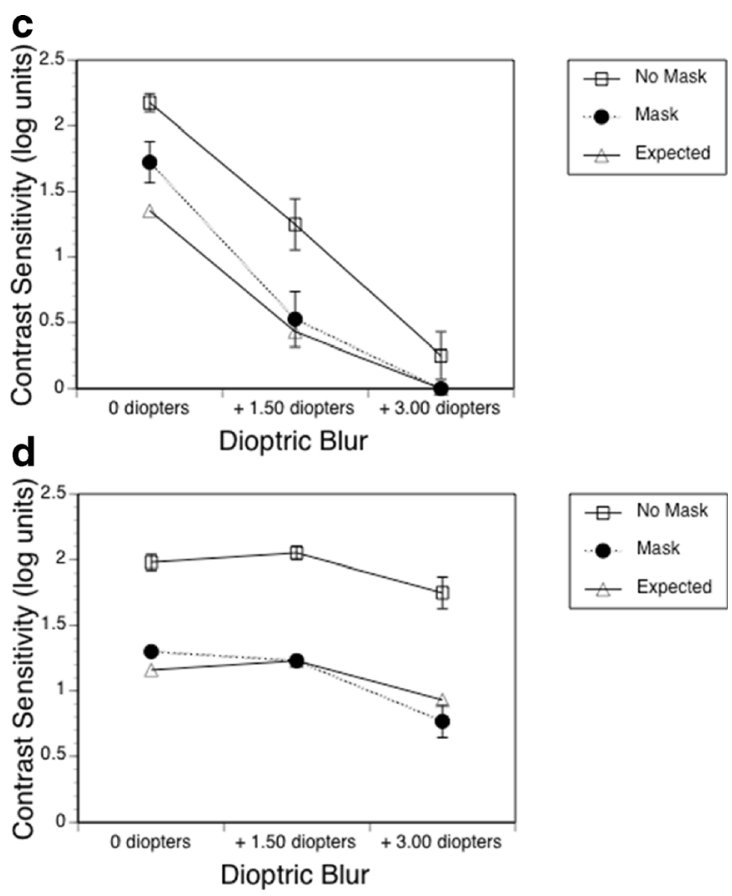

in parentheses. (c, d) Letter contrast sensitivities without (empty squares) and with (black circles) the semitransparent mask for each of the three levels of dioptric blur, measured at viewing distances of (c) $4 \mathrm{~m}$ and (d) $0.5 \mathrm{~m}$. The expected values based on a contrast reduction of $15 \%$ (decrement of $0.82 \log C S$ ) are indicated by the empty triangles. Error bars represent standard errors of the means

conditions were blocked and counterbalanced across subjects. The entire experiment lasted about $30 \mathrm{~min}$.

\section{Results}

The direction of the gaze cone was computed as the average of all adjustments in the centering task, expressed in degrees from the subject's straight-ahead. The width of the gaze cone was the unsigned sum of the two matching decentering values (to the left and to the right) for any given level of head orientation, mask, and diopter value. Thus, the cone width amounted to the angular difference between the leftward and rightward boundaries of the sector within which gaze directions were considered as looking at the subject.

Direction of the gaze cone We conducted a $3 \times 2 \times 3$ repeated measures analysis of variance (ANOVA) on the gaze cone's direction, with the factors Head Rotation $\left(-8^{\circ}, 0^{\circ}\right.$, or $\left.8^{\circ}\right)$, Mask (transparency $100 \%$ or $15 \%$ ), and Dioptric Blur (0, 1.5 , or 3 diopters). Degrees of freedom were GreenhouseGeisser corrected to account for a lack of sphericity. We found a significant main effect of head rotation (see Fig. 3): $F(1.27$, $29.17)=186.13, p<.001, \eta_{\mathrm{p}}{ }^{2}=.89$. When the head was rotated to its right, subjects adjusted the eyes to the right side of the actual center (by $-2.4^{\circ}$, on average). We describe this 


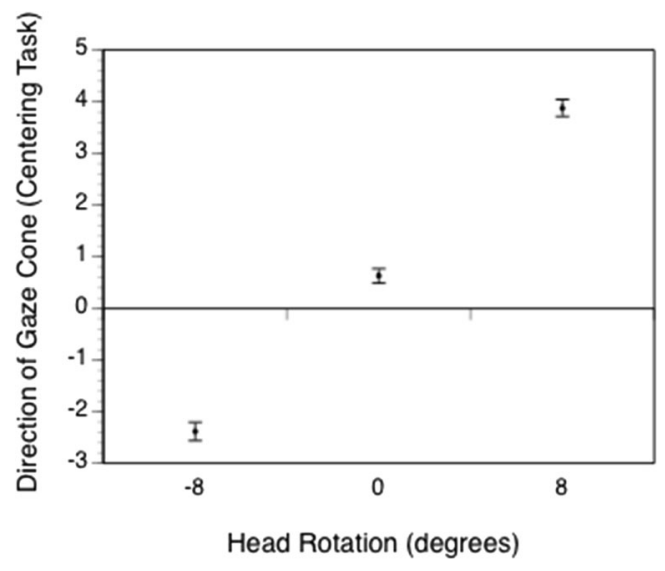

Fig. 3 Mean eye adjustment errors (in degrees) for the direction of the gaze cone as a function of head rotation. Negative head rotation values indicate a rotation to the right (clockwise yaw of the virtual head), positive values a rotation to the left. A value of $0^{\circ}$ means that the head was centered. Error bars represent standard errors of the means

effect as the gaze being attracted by the observer; one might also want to think about the effect as the virtual head repulsing the perceived gaze direction. ${ }^{1}$ When the head was rotated to the left (counterclockwise yaw), this effect was even larger: Subjects adjusted the eyes - which were actually looking directly at them-to $3.9^{\circ}$ on average, which is almost half the distance between the head rotation of $8^{\circ}$ and the actual center at $0^{\circ}$. When the head orientation was centered directly toward the subject, subjects adjusted the eyes practically without bias, to $0.6^{\circ}$ on average. Neither the mask nor dioptric blur produced a significant main effect. However, we did find a significant interaction between head rotation and mask (see Fig. 4): $F(1.95,44.77)=4.22, p=.022, \eta_{\mathrm{p}}{ }^{2}=.16$. Adding the mask to the head increased the effect of head rotation.

A separate ANOVA was conducted on the standard deviations of the gaze-centering data. Trials with the mask were associated with greater variability in the gaze direction judgments $\left(M=2.89^{\circ}\right.$ without mask vs. $M=3.29^{\circ}$ with mask): $F(1.00,23.00)=1.99, p=.009$.

Width of the gaze cone We conducted a $3 \times 2 \times 3$ repeated measures ANOVA on the gaze cone's width, using the same factors as before. We found a small, but significant, main effect of dioptric blur value (see Fig. 5): $F(1.96,45.08)=$ $5.18, p=.01, \eta_{\mathrm{p}}^{2}=.18$. The higher the level of dioptric blur,

${ }^{1}$ Note that the eccentricity of the eye within the head (eye socket) appears to determine the subjective gaze direction. Thus, since the pupil of the virtual head had to approach the rightmost edge of the eye socket (as seen by the subject) in order to be directed at the subject, the gaze looked more rightward than it actually was. This could be thought of as the virtual head repulsing the gaze away from its midline (direction of the nose). For a nice elaboration of the eccentricity account, see Todorović (2009).

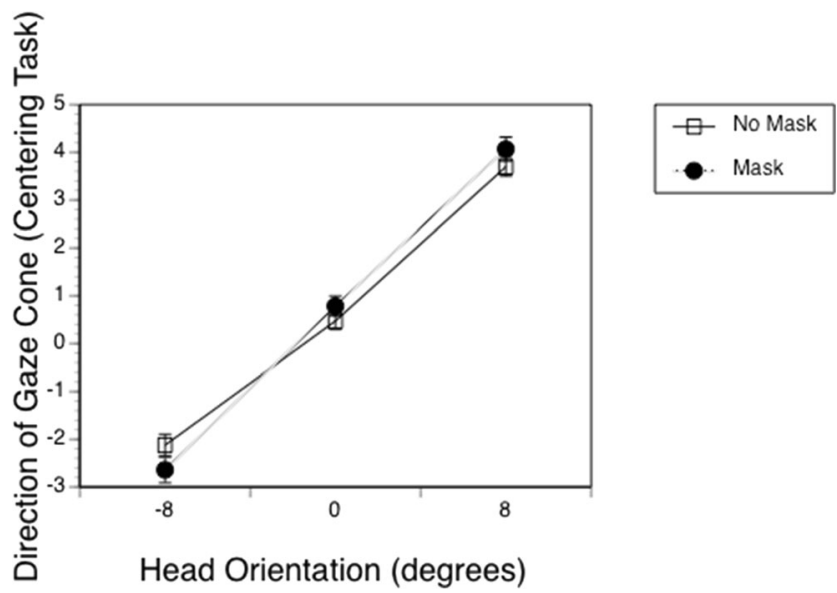

Fig. 4 Mean eye adjustments for the direction of the gaze cone as a function of head rotation, grouped by the Mask factor. Error bars represent standard errors of the means

the smaller the width of the gaze cone $\left(12.9^{\circ}\right.$ at 0 diopters, $11.7^{\circ}$ for 1.5 diopters, and $11.3^{\circ}$ for 3 diopters). The main effect of mask was not significant $[F(1.00,23.00)=0.64, p$ $\left.=.43, \eta_{\mathrm{p}}{ }^{2}=.03\right]$, nor was the interaction between mask and blur $\left[F(1.51,34.82)=2.46, p=.11, \eta_{\mathrm{p}}^{2}=.1\right]$. Head orientation did not significantly affect the width of the gaze cone.

\section{Discussion}

Inducing dioptric blur did not affect judgments of the direction of gaze. It did, however, affect the width of the gaze cone: The cone became narrower as the level of blur increased. This effect might be counterintuitive, since the reduction in visual acuity created by the blurring lenses should increase uncertainty. Subjects seemed to tighten their criterion for mutual gaze in the face of a less crisp image.

The degradation caused by the virtual mask increased the attractor effect of the head. This can be taken as a strategy shift in reaction to the poorer stimulus quality, which is very intuitive. Head orientation is factored into the judgments to a greater extent as the high-spatial-frequency information of

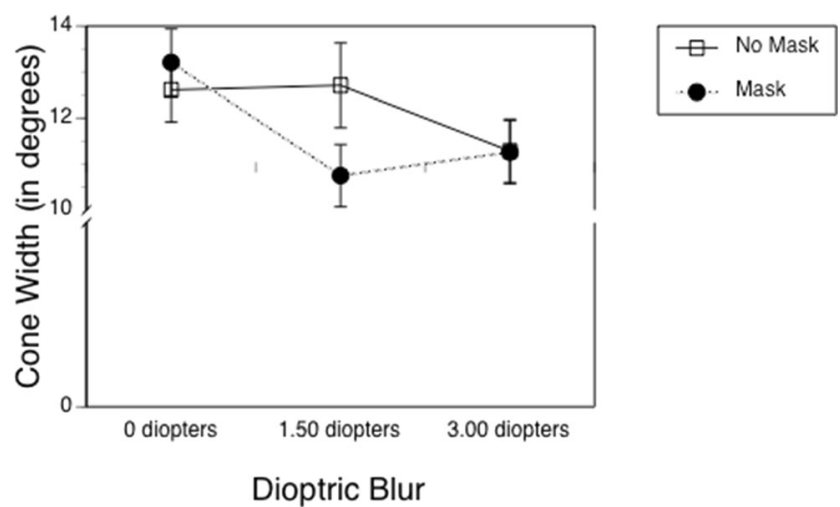

Fig. 5 Mean eye adjustments for the width of the gaze cone as a function of the three levels of dioptric blur, grouped by the Mask factor. Error bars represent standard errors of the means 
the looker's eyes is being reduced. Thus, coarser information replaces the fine-grained information that is no longer available. The virtual mask also made the gaze direction judgments more variable, which reflects the higher uncertainty. It is remarkable, however, that on average, the effects were very small indeed. Gaze direction was not seriously affected by the impairments brought about by degrading the stimulus and the observer's acuity.

The subjects in Experiment 1 were young (19-36 years) normally sighted subjects. In Experiment 2, we extended our investigation to include both young and older normally sighted subjects. We also extended our experimental paradigm to include measurements of mutual gaze in the vertical plane, because gaze direction in the real world is judged not only when people look from the left or the right, but also when they look upward or downward (depending on height differences between the looker and the subject).

\section{Experiment 2: Simulated impairment in younger and older observers}

In Experiment 2, we used a diffusing filter commonly applied to simulate losses of visual acuity and contrast sensitivity, as might occur in patients with moderate cataracts - an agerelated ocular condition that impairs the ability to recognize faces and facial expressions (Elliott, Patla, \& Bullimore, 1997). In light of the findings from Experiment 1, we tested the hypothesis that this simulated vision impairment would increase variability in the performance of the gaze perception task and would reduce the gaze cone width. We also investigated the effects of age on mutual gaze perception and predicted that the effects of the simulated cataract would be greater in older than in younger subjects.

\section{Method}

This experiment was approved by the institutional review board at Schepens Eye Research Institute, where the data were collected. Voluntary, written informed consent was obtained from all subjects after a full explanation of the study procedures.

Subjects A group of 32 normally sighted subjects participated (14 men, 18 women; $23-87$ years of age, $M=55.66$, $S D=$ 20.51). All had normal or corrected-to-normal visual acuity $(M=0.04 \log$ MAR, $S D=0.08)$ and contrast sensitivity $(M=$ $1.88 \log$ units, $S D=0.14$ ).

Design and procedure The study consisted of one session and took approximately 1 to $2 \mathrm{~h}$, including recording the vision measures and two blocks of the gaze cone task. One block was performed with the subject's normal or corrected- to-normal vision - that is, if necessary with the spectacles normally used in social situations when interacting with people at about a 1-m distance. The other block was performed while wearing lightweight goggles with an 0.3 opacity Bangerter diffusing filter (Fresnel Prism \& Lens Co., Eden Prairie, $\mathrm{MN}$ ) in front of each eye (and over the habitual spectacles, where these were needed). These diffusing filters reduced both visual acuity and contrast sensitivity (see the Results below, as well as Bowers \& Reid, 1997; McCulloch et al., 2011; Pérez, Archer, \& Artal, 2010). The 0.3 opacity value was selected to provide a simulation of moderate cataracts (Wood \& Carberry, 2006). The order of blocks was counterbalanced.

Vision measures Vision measures were conducted binocularly. Visual acuity measurements were taken at a 1-m test distance (the distance at which the gaze cone measurements were performed) using the "Tumbling E" option of the FrACT program (http://michaelbach.de/fract/html), on a NEC MultiSync LCD 2090UXi high-resolution monitor $(1,600 \times$ 1,200 at $85 \mathrm{~Hz}$ ) with an average luminance of $280 \mathrm{~cd} / \mathrm{m}^{2}$, controlled by a Cyperpower PC (Bach, 1996, 2006). Contrast sensitivity was assessed at a $450-\mathrm{mm}$ testing distance using a custom computerized program, equivalent to the PelliRobson chart, for letters subtending $2.5^{\circ}$ of visual angle.

Gaze cone task Subjects were seated $1 \mathrm{~m}$ from a computer monitor displaying the same 2-D virtual head as in Experiment 1 (but sized appropriately for the longer, 1-m viewing distance). A chinrest was used to align the subject to the eye level of the virtual head target and to maintain the 1-m testing distance. All subjects were presented with a virtual head that was aligned with the subject, was turned away by $8^{\circ}$ to the left or the right for measurements of the horizontal gaze cone, or was turned away $8^{\circ}$ up or down for measurements of the vertical gaze cone. As in Experiment 1, the subjects received instructions to perform one of four tasks: centering from an initial gaze to the left/right or up/down, and decentering the eyes of the virtual head toward the left/right or up/down. Subjects used the computer mouse to interactively adjust the eyes. In total, 24 trials were presented for the centering task and 24 for the decentering task, randomly interleaved.

\section{Results}

The data of one subject were removed because the vertical and horizontal gaze cone sizes exceeded four standard deviations from those for the remaining sample, suggesting that this individual failed to understand the instructions. To look at the effect of age on our measures, we conducted a median split ( $M d n=59$ years $)$ of the subjects into two age groups: an older group ( $M=71$ years, $S D=9, n=16)$ and a younger group ( $M$ $=36$ years, $S D=12, n=15$ ). An alpha level of .05 was used for all statistical tests. 
Vision measures Visual acuity and contrast sensitivity were subjected to a $2 \times 2$ repeated measures ANOVA with Vision (no filters vs. diffusing filters) and Age Group (older vs. younger) as factors. As expected, we found that the diffusing filters significantly reduced both visual acuity $[F(1,29)=198.82, p$ $\left.<.001, \eta_{\mathrm{p}}{ }^{2}=.87\right]$ and contrast sensitivity $[F(1,28)=57.07, p$ $<.001, \eta_{\mathrm{p}}{ }^{2}=.67$ ] (Fig. 6). In addition, we found a significant main effect of age group for both visual acuity $[F(1,29)=$ $\left.5.16, p=.03, \eta_{\mathrm{p}}{ }^{2}=.15\right)$ and contrast sensitivity $[F(1,28)=$ $\left.7.80, p=.009, \eta_{\mathrm{p}}{ }^{2}=.22\right]$, with better acuity and contrast sensitivity in the younger than in the older group. Finally, a marginally significant interaction emerged between vision and age group for both visual acuity $\left[F(1,29)=3.91, p=.048, \eta_{\mathrm{p}}{ }^{2}\right.$ $=.13]$ and contrast sensitivity $\left[F(1,28)=5.10, p=.032, \eta_{\mathrm{p}}{ }^{2}=\right.$ .15]. Specifically, the diffusing filters had a greater negative effect on the visual acuity and contrast sensitivity of the older than of the younger group (see Fig. 6).

Horizontal direction of the gaze cone We conducted a $2 \times 2$ $\times 3$ repeated measures ANOVA on the horizontal direction of the gaze cone. The factors were Age Group (older vs. younger), Vision (no filters vs. diffusing filters), and Head Rotation $\left(-8^{\circ}, 0^{\circ}\right.$, or $\left.8^{\circ}\right)$. As in Experiment 1 , we found a significant main effect of head rotation: $F(2,56)=64.44, p<.001, \eta_{\mathrm{p}}{ }^{2}=$ .70. As can be seen in Fig. 7, the perceived horizontal direction of the gaze cone shifted toward the direction in which the virtual head was oriented (attractor effect of head orientation). This effect was robust for both the younger and the older observers. The analysis also revealed a significant main effect of vision $\left[F(1,28)=6.52, p=.02, \eta_{\mathrm{p}}{ }^{2}=.19\right]$; the overall head orientation effect was less with than without the filter. In addition, we observed a Vision $\times$ Age Group interaction effect approaching significance: $F(1,28)=3.95, p=.057, \eta_{\mathrm{p}}{ }^{2}=.12$. Specifically, the attractor effects of head orientation were similar with and without the filter for the younger group, but decreased with the filter for the older group (Fig. 7). There were no other significant main effects or interactions.

The same analysis was performed on the variability (i.e., standard deviation) of the horizontal direction of the gaze cone. Although no significant main effect of vision was apparent (no

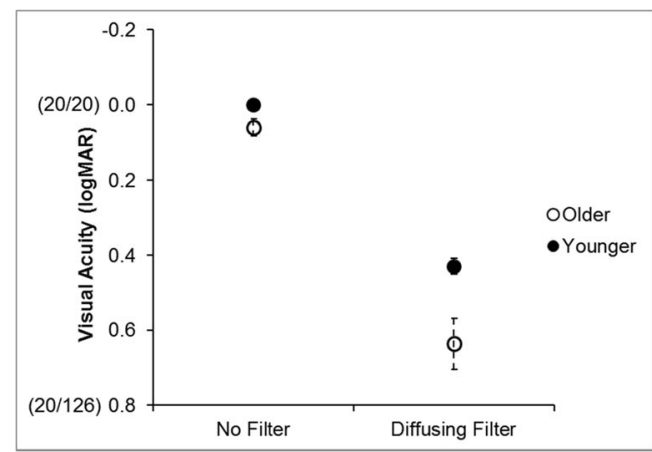

Fig. 6 Mean visual acuity (left) and contrast sensitivity (right) of the subjects in the older and younger groups without and with the diffusing filters. Values in parentheses on the $y$-axis in the left panel are Snellen acuities. Error bars represent standard errors of the means

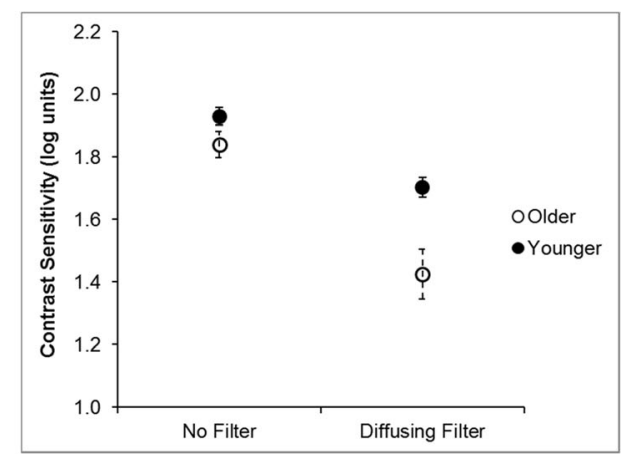

filters vs. diffusing filters), the ANOVA yielded a main effect of head rotation $\left[F(2,56)=5.85, p=.005, \eta_{\mathrm{p}}{ }^{2}=.17\right]$, such that the variability was greater when the virtual head was rotated right than when it was centered or rotated left. In addition, the main effect of age group was significant $[F(1,28)=9.93, p=.004$, $\left.\eta_{\mathrm{p}}{ }^{2}=.26\right]$, indicating that the older age group had more variability in their perceived direction of the gaze cone than did the younger group. However, we did find an interaction between head rotation and age group $\left[F(2,56)=4.04, p=.02, \eta_{\mathrm{p}}{ }^{2}=\right.$ $.13]$; thus, the significant difference between the two groups was due to the centered and right head rotations (Fig. 8).

Vertical direction of the gaze cone The data for the vertical direction of the gaze cone were analyzed with the same $2 \times 2 \times$ 3 ANOVA as the horizontal data. The orientation of the virtual head had a significant effect on the perceived vertical direction of gaze: $F(2,56)=60.64, p<.001, \eta_{\mathrm{p}}^{2}=.68$. As in the horizontal direction, the subjects' perception of the direction of gaze shifted toward the vertical rotation of the virtual head (Fig. 9). The analysis also showed a significant main effect of age group $\left[F(1,28)=86.82, p=.003, \eta_{\mathrm{p}}^{2}=.28\right]$, indicating that the older group tended to judge the direction of mutual gaze as being more upward than did the younger group, regardless of the orientation of the virtual head (Fig. 9). No other significant main effects emerged (no effect of the filters), and no significant interactions.

The same analysis performed on the variability (i.e., standard deviation) of the vertical direction of the gaze cone yielded only a main effect of age group: $F(1,28)=8.56, p=$ $.007, \eta_{\mathrm{p}}{ }^{2}=.23$. In the vertical direction, the older group had more variability in their judgments of gaze $\left(M=3.32^{\circ}, S D=\right.$ $1.62)$ than did the younger group $\left(M=2.06^{\circ}, S D=1.62\right)$.

Horizontal width of gaze cone A $2 \times 2 \times 2$ repeated measures ANOVA with Age Group (older vs. younger), Vision (no filters vs. diffusing filters), and Head Orientation (centered vs. angled) as factors was conducted on the horizontal width of the gaze cone. Replicating Experiment 1, we found the main effect of vision to be significant $\left[F(1,28)=4.32, p=.047, \eta_{\mathrm{p}}{ }^{2}\right.$ $=.13]$, indicating that the horizontal gaze cone width was 
Older

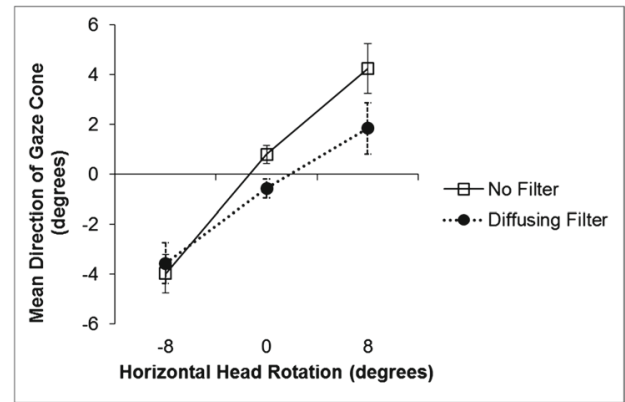

Fig. 7 Mean eye adjustments for the direction of the gaze cone, by the older (left) and the younger (right) age groups, as a function of horizontal head rotations without and with the diffusing filters. Negative values

smaller when the subjects wore the filters than when they did not (see Fig. 10). There was also a significant main effect of head orientation $\left[F(1,28)=9.50, p=.005, \eta_{\mathrm{p}}{ }^{2}=.25\right]$, such that the gaze cone was wider when the virtual head was centered than when it was rotated to the right or the left (see Fig. 10). No other significant main effects or interactions were apparent.

Vertical width of gaze cone The same $2 \times 2 \times 2$ repeated measures ANOVA performed on the horizontal gaze cone width data was used to analyze the vertical gaze cone width. As with the horizontal gaze cone, the vertical gaze cone was significantly wider $\left[F(1,28)=24.50, p<.001, \eta_{\mathrm{p}}{ }^{2}=.47\right]$ when the virtual head was centered $\left(M=12.44^{\circ}, S D=6.51\right)$ than when it was rotated up or down $\left(M=10.11^{\circ}, S D=6.58\right)$. No other significant main effects or interactions emerged.

\section{Discussion}

Simulation of cataracts via Bangerter diffusing filters, which reduced visual acuity and contrast sensitivity, affected

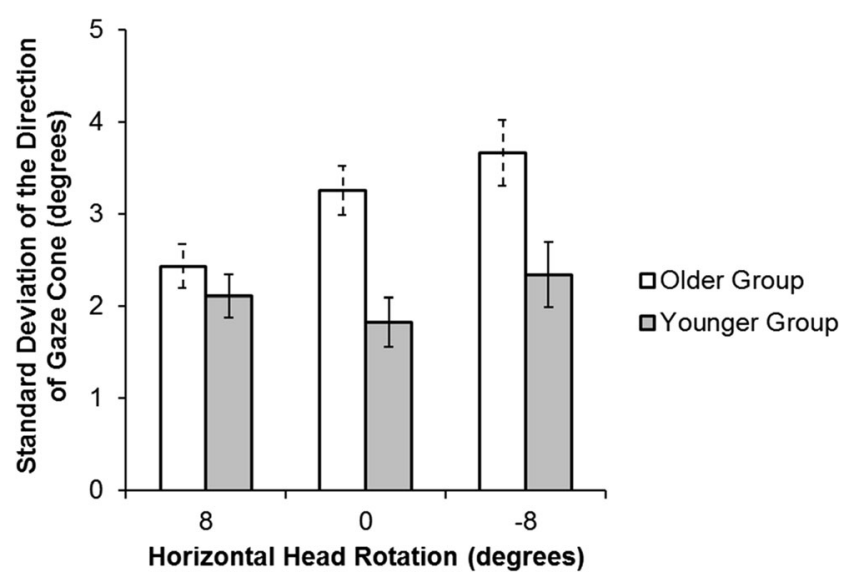

Fig. 8 Means of the standard deviations (i.e., variability) of the average eye adjustments for the direction of the gaze cone as a function of horizontal head rotations for the subjects in the older and younger groups. Error bars represent standard errors of the means
Younger

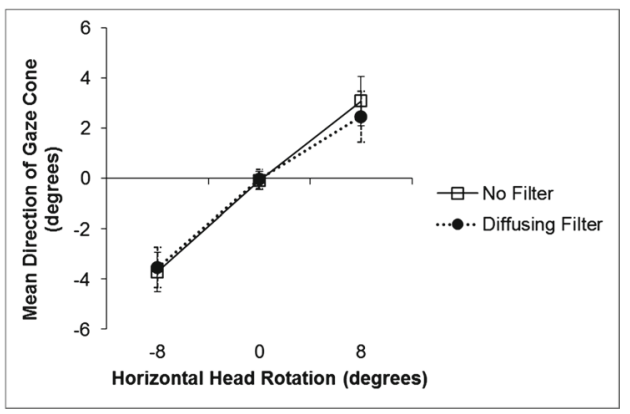

indicate a rotation to the right, positive values a rotation to the left. A value of $0^{\circ}$ means that the head was centered. Error bars represent standard errors of the means

judgments of the direction and width of the horizontal gaze cone. As in Experiment 1, the width of the horizontal gaze cone was narrower when the subjects' vision was reduced. However, unlike in the virtual-mask condition in Experiment 1 , the attractor effect of the head on perceived gaze direction did not increase with the filters. This difference may have occurred because the Bangerter diffusing filters did not degrade vision as much as the virtual semitransparent white mask.

We extended the paradigm in Experiment 2 to include trials performed in the vertical (up and down) plane in addition to the horizontal (right and left) plane. Unlike the horizontal gaze cone, the vertical gaze cone parameters were not affected by the simulated vision impairment. However, we found that the orientation of the virtual head attracted the mean gaze direction toward the direction that the head was rotated, regardless of whether the head rotation was horizontal or vertical.

In Experiment 2, we widened our population sample (2387 years) to investigate the effects of aging on the gaze cone

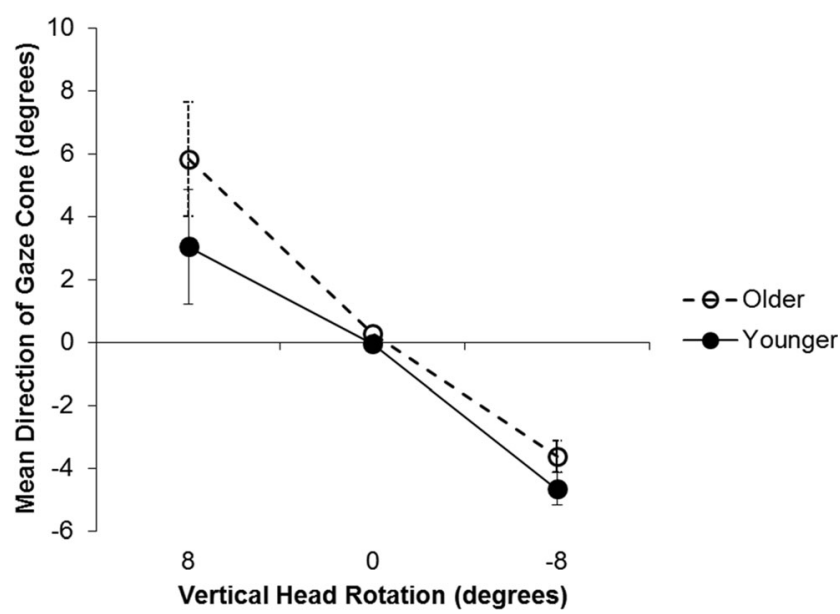

Fig. 9 Mean eye adjustments for the direction of the gaze cone as a function of vertical head rotations. Negative values indicate a downward rotation, positive values an upward rotation. A value of $0^{\circ}$ means that the head was centered. Error bars represent standard errors of the means 


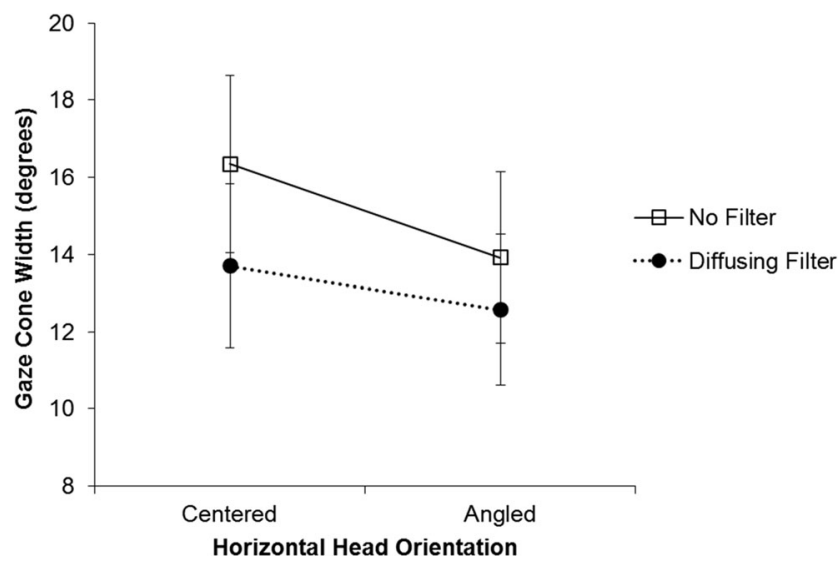

Fig. 10 Mean eye adjustments for the width of the gaze cone without and with diffusing filters when the virtual head was centered versus when it was angled right or left. Error bars represent standard errors of the means

parameters. We found that the gaze cone width was unaffected by the age of the subject. The directions of mutual gaze, on the other hand, differed significantly between the younger and older subjects. The younger subjects were more consistent (i.e., had less variability) in their judgments of horizontal and vertical gaze direction. Furthermore, in the vertical direction, the younger group tended to judge the mean direction of gaze as being lower than did the older group.

\section{General discussion}

Under optimal viewing conditions, we take a large range of gaze directions to constitute mutual gaze. This range of tolerance, the cone of gaze, is typically about $10^{\circ}$ when using a human looker, and it can be considerably larger when using a virtual head whose facial expression is changed to express anger, or when the subject is anxious (Harbort, Witthöft, Spiegel, Nick, \& Hecht, 2013). But what happens when stimulus uncertainty is increased by suboptimal viewing conditions, such as low light levels, refractive blur, or the onset of age-related ocular disease? Does the large tolerance range in the normal cone of gaze have the advantage of allowing for the maintenance of perceptual and communicative skills in the face of stimulus degradation or visual impairment?

To address these questions, we conducted two experiments to investigate how the perceived center of gaze and the perceived cone of gaze would change when visual acuity and contrast sensitivity were reduced. The results suggest that perceived gaze direction tends to be very robust. The width of the cone of gaze, however, was more readily affected by simulated visual impairment. Interestingly, the increase of uncertainty that accompanied the blur did not lead to a more relaxed criterion for mutual gaze, but rather to a tightened criterion; in both experiments, the width of the horizontal cone of gaze was reduced as uncertainty increased. Thus, subjects behaved as if they required a given degree of certainty to decide that mutual gaze was established.

It should be noted that, in direct contrast to our findings, Mareschal, Calder, and Clifford (2013) found gaze discrimination thresholds to be higher when noise was added to the eye region of synthetic face stimuli. This finding suggests that gaze is more likely to be taken as being directed at the observer whenever uncertainty is introduced. Within our paradigm, such a bias should have resulted in a wider gaze cone with increasing uncertainty. We propose that this difference in the results may be attributed to the differing methodologies. Whereas our subjects made direct interactive assessments of mutual gaze, which are somewhat similar to deliberate gaze judgments in everyday situations, Mareschal, Calder, and Clifford's subjects had to make mutual gaze judgments of briefly $(400 \mathrm{~ms})$ presented pictures of faces by means of a forced choice task. It is possible that we did not replicate the previous results because only the eyes of the earlier stimuli were blurred, whereas we manipulated the entire image. However, it is more likely that the method of assessment is the critical factor underlying the difference.

The results of Experiment 1 suggest that, in addition to altering the criterion for accepting mutual gaze, subjects may change their strategy for judging gaze direction when access to visual information is limited. In both of our experiments, the direction of the head attracted the central direction of the gaze cone. However, when a virtual semitransparent mask degraded the stimulus (Exp. 1), this effect became stronger. Thus, it appears that when eye position became more difficult to discern, subjects adopted a strategy of judging mutual gaze that relied more heavily on head orientation than on eye position. This makes sense because head position is carried by lower spatial frequencies, and would, therefore, be less affected by the stimulus degradation. However, neither dioptric blur (Exp. 1) nor Bangerter diffusing filters (Exp. 2) increased the effect of the head rotation on the central direction of the gaze cone, possibly because in these simulations the visibility of the virtual eyes was degraded to a lesser extent than with the virtual mask.

In Experiment 2, we investigated the effects of age and vision impairment on mutual gaze perception. Overall, the gaze judgments of the older group were fairly similar to those of the younger group. The main difference was greater variability in the judgments of gaze direction in the older group, but there was little effect of age on the perceived width of the gaze cone. Contrary to our prediction that older subjects would be more strongly affected by the simulated impairments than would the younger subjects, only one interaction between vision (no filter or diffusing filter) and age group (young or old) approached significance. This may seem surprising, given that the filters reduced visual acuity and contrast sensitivity to a greater extent in the older than in the younger subjects. However, one interpretation of these findings is that mutual 
gaze perception is relatively robust in the face of increasing age and mild-to-moderate levels of vision impairment.

In this study, we used simulations of vision impairments that might be experienced by normally sighted people when wearing an incorrect spectacle prescription or by people with central vision loss due to cataracts (or any other condition that reduces visual acuity and contrast sensitivity). We previously found that patients with central vision loss that included a central scotoma (a blind area in central vision) had significantly more variable gaze direction judgments than did age-matched normally sighted subjects (Sheldon, Quint, Hecht, \& Bowers, 2014). Our present simulations produced similar results in healthy subjects. This is compatible with findings by Geringswald, Baumgartner, and Pollmann (2012), who simulated the effects of a central scotoma in healthy observers using a gaze-contingent stimulus presentation. In a complex visual search task involving contextual cueing, the artificial scotoma reduced the facilitation effect of stimulus repetition. Thus, even complex visual processes that leave room for compensation were negatively affected by the scotoma. Surprisingly, apart from the increased variability, the central vision loss affected our patients to a remarkably small extent as they judged mutual gaze (Sheldon et al., 2014). In the present study, the healthy subjects appeared to compensate for the impairment by tightening their criterion for mutual gaze when confronted with dioptric blur or a diffusing filter, which could point to a process by which patients adapt to their visual impairment and are able to achieve close-to-normal performance with regard to higher-order visual perception even though their acuity is compromised.

Simulations offer the advantage of allowing us to conduct within-subjects analyses of the effects of different levels of impairment, which can only be studied between groups when real vision impairment is involved. However, simulations do not reproduce all of the characteristics of real vision impairment, and importantly, the responses of normally sighted observers who are exposed to simulations for only a short period of time might not reflect the same compensatory strategies that develop as patients adapt to their vision impairment over a longer period of time.

In summary, it cannot be taken for granted that the effects of aging and visual impairment on the judgment of gaze direction and mutual gaze amount to a straightforward loss in performance. Our results suggest that compensation mechanisms and criterion changes take place that allow us to perform better than would be predicted by a simple extrapolation from the losses in basic acuity and contrast sensitivity.

Author note Agnes Münch programmed the gaze cone task. This study was supported in part by NIH Grant No. T35EY007149 and by an Alice J. Adler Fellowship from the Schepens Eye Research Institute.

\section{References}

Ando, S. (2002). Luminance-induced shift in the apparent direction of gaze. Perception, 31, 657-674. doi:10.1068/P3332

Bach, M. (1996). The Freiburg Visual Acuity Test-Automatic measurement of visual acuity. Optometry \& Vision Science, 73, 49-53. doi: 10.1097/00006324-199601000-00008

Bach, M. (2006). The Freiburg Visual Acuity Test—Variability unchanged by post-hoc re-analysis. Graefe's Archive for Clinical and Experimental Ophthalmology, 245, 965-971. doi:10.1007/s00417-006-0474-4

Bowers, A. R., \& Reid, V. M. (1997). Eye movements and reading with simulated visual impairment. Ophthalmic and Physiological Optics, 17, 392-402. doi:10.1046/j.1475-1313.1997.97000161.x

Elliott, D. B., Patla, A., \& Bullimore, M. A. (1997). Improvements in clinical and functional vision and perceived visual disability after first and second eye cataract surgery. British Journal of Ophthalmology, 80, 799-804. doi:10.1136/bjo.81.10.889

Gamer, M., \& Hecht, H. (2007). Are you looking at me? Measuring the cone of gaze. Journal of Experimental Psychology, 33, 705-715. doi:10.1037/0096-1523.33.3.705

Gamer, M., Hecht, H., Seipp, N., \& Hiller, W. (2011). Who is looking at me? The cone of gaze widens in social phobia. Cognition and Emotion, 25, 756-764. doi:10.1037/0096-1523.33.3.705

Geringswald, F., Baumgartner, F., \& Pollmann, S. (2012). Simulated loss of foveal vision eliminates visual search advantage in repeated displays. Frontiers in Human Neuroscience, 6, 134. doi:10.3389/ fnhum.2012.00134

Gibson, J. J., \& Pick, A. D. (1963). Perception of another person's looking behavior. American Journal of Psychology, 76, 386-394. doi:10.2307/1419779

Harbort, M., Witthöft, M., Spiegel, J., Nick, K., \& Hecht, H. (2013). The widening of the gaze cone in patients with social anxiety disorder and its normalization after CBT. Behaviour Research and Therapy, 51, 359-367. doi:10.1016/j.brat.2013.03.009

Hecht, H., Weiland, R., \& Boyarskaya, E. (2011). The cone of gaze. In H. Koshimizu \& B. M. Wilamowski (Eds.), 4th International IEEE Conference on Human System Interactions (HSI 2011) (pp. 378385). Piscataway, NJ: IEEE Press. doi:10.1109/HSI.2011.5937396

Kleinke, C. L. (1986). Gaze and eye contact: A research review. Psychological Bulletin, 100, 78-100. doi:10.1037/0033-2909.100.1.78

Mareschal, I., Calder, A. J., \& Clifford, C. W. G. (2013). Humans have an expectation that gaze is directed toward them. Current Biology, 23, 717-721. doi:10.1016/j.cub.2013.03.030

McCulloch, D. L., Loffler, G., Colquhoun, K., Bruce, N., Dutton, G. N., $\&$ Bach, M. (2011). The effects of visual degradation on face discrimination. Ophthalmic and Physiological Optics, 31, 240-248. doi:10.1111/j.1475-1313.2011.00828.x

Pelli, D. G., Robson, J. G., \& Wilkins, A. J. (1988). The design of a new letter chart for measuring contrast sensitivity. Clinical Vision Science, 2, 187-199.

Pérez, G. M., Archer, S. M., \& Artal, P. (2010). Optical characterization of Bangerter foils. Investigative Ophthalmology \& Visual Science, 51, 609-613. doi:10.1167/iovs.09-3726

Sheldon, S., Quint, J., Hecht, H., \& Bowers, A. R. (2014). The effect of central vision loss on perception of mutual gaze. Optometry and Vision Science, 91, 1000-1011. doi:10.1097/OPX. 0000000000000314

Todorović, D. (2009). The effect of face eccentricity on the perception of gaze direction. Perception, 38, 109-132. doi:10.1068/p5930

Wood, J. M., \& Carberry, T. P. (2006). Bilateral cataract surgery and driving performance. British Journal of Ophthalmology, 90, 12771280. doi:10.1136/bjo.2006.096057 